\title{
Streifen auf dem Nagel: Wann besteht Melanomverdacht?
}

\begin{abstract}
Hochauflösende Dermatoskopie-Aufnahmen haben europäische Dermatologen retrospektiv ausgewertet, um zu prüfen, wie gut sich ein Melanom bei streifenförmiger Nagelpigmentierung dermatoskopisch diagnostizieren lässt.
\end{abstract}

$\mathrm{D}$ ie untersuchten Bilder stammten von 82 erwachsenen Patienten. Bei 25 Patienten wurde nach histopathologischer Untersuchung ein Nagelmelanom festgestellt, bei 57 Patienten eine benigne Läsion, von denen 32 melanozytäre Nävi und 25 eine benigne melanozytäre Hyperplasie hatten.

Unter den 25 Patienten mit einem Melanom war bei elf Patienten das Malignom invasiv (mittlere Breslow-Dicke $0,87 \mathrm{~mm}$ ). Die meisten Läsionen betrafen Fingernägel, und zwar knapp $70 \%$ der benignen Läsionen und $80 \%$ der Melanome. Ein einzelner Streifen im Nagel wurde bei elf Patienten (19,3\%) mit benigner Veränderung dokumentiert, aber bei keinem Patienten mit Melanom.
Die univariate Regressionsanalyse lieferte mehrere Parameter, mit denen sich ein Melanom diagnostizieren ließ; Ausbreitung über mindestens zwei Drittel der Nagelfläche, graue oder schwarze Farbe, keine homogenen Farben und Dicken der Streifen eines Nagels, granuläre Pigmentierung sowie Hutchinson- und MikroHutchinson-Zeichen. Die Odds Ratio (OR) bei Analyse der Farbe errechnete sich für Grau zu 14,88. Bei Nachweis von Hutchinson- und Mikro-HutchinsonZeichen lag die OR bei 56,1, bei einer Schwarzfärbung bei 12 .

Nach der multivariaten Analyse blieben nur noch zwei Parameter mit signifikantem Vorhersagewert übrig: zum einen die Ausbreitung der Streifen über mehr als zwei Drittel der Nagelfläche mit einer OR von 8,14 . Nur bei $44 \%$ der Patienten mit benigner Läsion war diese über mindestens zwei Drittel der Nagelfläche ausgebreitet. Dagegen war das bei allen Patienten mit einem Melanom der Fall.

Zweiter Parameter war die Farbe Grau oder Schwarz mit einer OR von 9,70. Bei Patienten mit benigner Läsion waren graue oder schwarze Streifen bei 17,5\% und 5,3\% nachweisbar. Mit einem Anteil von $76 \%$ und $40 \%$ war der Anteil der betroffenen Patienten in der Melanomgruppe dagegen signifikant höher.

Fazit: Bei Erwachsenen mit Melanonychia striata longitudinalis gibt es mehrere klinisch oder dermatoskopisch erkennbare Merkmale, die Hinweise für ein Melanom als Ursache sein können: Ausbreitung über mindestens zwei Drittel des Nagels, graue oder schwarze Farbe und eine irreguläre braune, granuläre Pigmentierung mit oder ohne Nageldystrophie.

Peter Leiner

Benati E et al. Clinical and dermoscopic clues to differentiate pigmented nail bands: an International Dermoscopy Society study. J Eur Acad Dermatol Venereol 2017; 31: 732-6

\section{Infliximab wirkt rasch gegen Psoriasis}

\section{In einer Vergleichsstudie haben Patienten mit Psoriasis unter Infliximab rascher einen PASI 75 erreicht als unter Etanercept. Längerfristig war aber kein Unterschied im Therapieerfolg festzustellen.}

$\mathrm{N}$ iederländische Hautärzte haben in einer Studie die Wirkmuster von Infliximab und Etanercept mit 48 Patienten getestet, die an mäßiger bis schwerer Psoriasis litten. 25 erhielten den TNF-Blocker Infliximab $5 \mathrm{mg}$ i.v. in den Wochen $0,2,6,14$ und 22. Die übrigen 23 bekamen zweimal wöchentlich $50 \mathrm{mg}$ Etanercept s.c., was dem Doppelten der üblicherweise empfohlenen Dosis entspricht.

Primärer Studienendpunkt war das Erreichen von PASI 75 nach 24 Wochen. Das gelang bei $72 \%$ der Patienten unter Infliximab und bei $35 \%$ unter Etanercept. PASI 75 nach Woche 6 war bei $52 \%$ der Infliximab- und bei $4 \%$ der Etanercept-Patienten zu verzeichnen. Die PASI75-Ergebnisse nach Woche 12 lagen bei $76 \%$ (Infliximab) und 22\% (Etanercept).
Weniger ausgeprägt waren die Unterschiede in der subjektiven Einschätzung. $88 \%$ der mit Infliximab behandelten Patienten gaben nach 24 Wochen an, ihr Hautbild sei gut oder vollkommen unter Kontrolle. In der Etanercept-Gruppe waren es $70 \%$. Auch die Remissionszeiten nach Absetzen der Studienmedikation differierten mit im Mittel 15,5 Wochen (Infliximab) gegenüber 13,7 Wochen (Etanercept) nicht signifikant.

22 Patienten, elf in jeder Gruppe, setzten die Therapie nach 24 Wochen fort. Nach Woche 48 lagen die PASI-75Anteile bei $67 \%$ für Infliximab und $50 \%$ für Etanercept. Die Differenz war statis-

Insgesamt kam es unter Infliximab zu mehr Nebenwirkungen, aber nur $5 \%$ watisch nicht mehr signifikant. ren schwerwiegender Natur. Vor allem Kreislaufstörungen und Abweichungen im Blutbild waren unter Infliximab häufiger. Die Quote schwerer Nebenwirkungen unter Etanercept betrug $7 \%$.

Fazit: Infliximab schneidet bei den kurzzeitigen Effekten besser ab als Etanercept. Langfristig gleicht sich die Wirkung aber aus. Die Autoren kommen so zu dem Schluss, dass beide Behandlungsformen effektiv und für Patienten mit mäßiger bis schwerer Psoriasis zu empfehlen sind. Diese Erkenntnisse wurden ohne finanzielle Unterstützung der Industrie gewonnen. Jedoch wurde die anvisierte Zahl von 120 rekrutierten Patienten weit verfehlt. Vor allem die Aussagen zu den Langzeiteffekten stehen statistisch auf dünnen Beinen.

Dr. Robert Bublak

De Vries ACQ et al. A prospective randomized controlled trial comparing infliximab and etanercept in patients with moderate-to-severe chronic plaque-type psoriasis: the Psoriasis Infliximab vs. Etanercept Comparison Evaluation (PIECE) study. Br J Dermatol 2017; 176: 624-633 